\title{
Novel associations between cytokines and pulmonary involvement in juvenile dermatomyositis - a cross sectional study of long-term disease
}

Henriette Marstein Msc ${ }^{1}$, Thomas Schwartz MD PhD ${ }^{1}$, Trond Mogens Aaløkken MD PhD ${ }^{3}$, May Britt Lund MD $\mathrm{PhD}^{6,4}$, Berit Flatø MD $\mathrm{PhD}^{2,4}$, Ivar Sjaastad MD PhD ${ }^{1,5}$, Helga Sanner MD $\mathrm{PhD}^{2,7}$

${ }^{1}$ Institute for Experimental Medical Research and KG Jebsen Center for Cardiac research, Oslo, Norway;

${ }^{2}$ Department of Rheumatology, Oslo University Hospital, Rikshospitalet, Oslo, Norway;

${ }^{3}$ Department of Radiology, Oslo University Hospital, Rikshospitalet, Oslo, Norway;

University Hospital and University of Oslo, Oslo, Norway

${ }^{4}$ Institute for Clinical Medicine, Medical Faculty, University of Oslo, Oslo, Norway

${ }^{5}$ Department of Cardiology, Oslo University Hospital Ullevål, Oslo, Norway

${ }^{6}$ Department of Respiratory Medicine, Oslo University Hospital, Rikshospitalet, Oslo, Norway

${ }^{7}$ Bjørknes University College, Oslo, Norway

Address correspondence to: Helga Sanner, MD, Department of Rheumatology, Oslo University Hospital, Rikshospitalet, 0027 Oslo, Norway.

Telephone: 004723070000

Fax: 004723072990

E-mail: helsan@ous-hf.no 
Key words: dermatomyositis, outcome assessment, respiratory function tests, high resolution computed tomography, case-control studies, cytokines 


\section{ABSTRACT}

Objectives: To examine associations between cytokines and pulmonary involvement in patients with medium- to long-term Juvenile Dermatomyositis (JDM).

Methods: In a cross-sectional study, 58 patients examined median(range) 16.8 (6.6-27.0) years after symptom onset were stratified in inactive (JDM-inactive) and active (JDM-active) disease (updated PRINTO criteria); 56 age/sex matched controls were included. Twenty-nine cytokines (in serum) were analysed (Luminex technology/ELISA). Pulmonary function test (PFT) included forced vital capacity (FVC), total lung capacity (TLC) and diffusing capacity for carbon monoxide (DLCO) reported as \% of predicted and low FVC/TLC/DLCO. In patients, the presence of clinical pulmonary damage was assessed and HRCT scans were scored for interstitial lung disease (ILD), chest wall calcinosis and airways disease.

Results: Median age of patients was 21(7-55) years, 59\% were female and 36\% inactive. In JDM-active and all patients, higher MCP-1, IP-10 and eotaxin correlated with HRCT findings $\left(\mathrm{r}_{\mathrm{s}}\right.$ 0.34-0.61; $\left.\mathrm{p}^{\mathrm{s}}<0.05\right)$. MCP-1 and eotaxin correlated with pulmonary damage in JDM-active and all patients $\left(\mathrm{r}_{\mathrm{s}}\right.$ 0.41-0.49; $\mathrm{p}$ s $\left.<0.01\right)$. Higher TGF- $\beta 1$ and PDGF (growth factors) were associated with lower lung volumes (FVC/TLC measures) in all patients; PDGF in JDM-active and TGF- $\beta 1$ in JDM-inactive patients. IP-10 correlated with TLC\% in JDM-active patients. No associations between cytokines and PFT were found in controls.

Conclusions: In JDM, we found a novel association (not previously described in myositis) between eotaxin and pulmonary involvement; we have previously shown an association between eotaxin and cardiac dysfunction. The associations between IP-10/growth factors/MCP-1 and pulmonary involvement are novel in JDM and were mostly seen in JDM-active patients.

\section{KEY MESSAGES:}


- After long-term follow-up in JDM, cytokines are upregulated and lung involvement (mostly subclinical) is common

- We found a novel association between eotaxin and pulmonary involvement (damage and structure).

- Associations between IP-10/growth factors/MCP-1 and pulmonary involvement were found (previously shown in adult onset IIM).

\section{INTRODUCTION:}

Juvenile Dermatomyositis (JDM) is the most common idiopathic inflammatory myopathy (IIM) of childhood. Vasculopathy and interferon (IFN) driven inflammation is thought to be central in the pathogenesis in this multiorgan disease [1] mainly characterized by skin and muscle involvement, but internal organs like lungs and heart can also be affected. Clinically manifest lung disease in JDM is rare $[1,2]$. However, studies have described reduced pulmonary function in JDM patients indicating lung involvement, especially in the Japanese population [3-6]. We have previously reported reduced pulmonary function tests (PFT) in JDM patients assessed after medium- to long-term follow-up; with reduced gas diffusing capacity and lung volumes in $49 \%$ and $25 \%$, respectively. Also, $37 \%$ had structural abnormalities detected by high resolution computed tomography (HRCT) [7]. Importantly, the lung findings were mostly subclinical and the underlying mechanisms are not known. On the other hand, interstitial lung disease (ILD) is a frequent complication in adult onset IIMs, including polymyositis (PM) and dermatomyositis (DM) and is associated with high morbidity and mortality [1, 8].

IFN`s are secreted from immune cells as a response to activated dendritic cells which are believed to be the molecular onset of JDM [9].Studies performed on both cohorts with variable disease duration and mixed patients with IIM (including DM, PM and JDM and JDM-cohorts, 
compared to controls shows fincreased levels of the IFN induced cytokines; interleukin (IL) 6 (IL-6), IL-10, interferon gamma-induced protein 10 (IP-10/CXCL10), monocyte chemoattractant protein-1 MCP-1, and macrophage inflammatory protein-1 (MIP-1 $\alpha$ and MIP-1 $\beta$ ) are reported in studies performed on small patient cohorts with variable disease duration and mixed patients with IIM (including DM, PM and JDM) compared to controls-[8-12]. These studies report associations between global disease activity and IL-6, IL-8, IP-10 and tumor necrosis factor alpha (TNF $\alpha)[8,10,13,14]$. Also in adult onset IIM, there are associations between ILD and Il6 , TNF $\alpha$, IP-10, MCP-1, transforming growth factor beta 1(TGF- $\beta 1$ ), and platelet derived growth factor (PDGF) $[8,10,13]$, indicating a role for cytokines influence upon lung involvement.

We have previously shown an increase in the circulating chemokines eotaxin, MCP-1 and IP-10 in JDM patients compared to controls [15], and an association between eotaxin, MCP-1 and both disease activity in muscle and skin (measured by global Disease Activity Score, DAS) and cardiac dysfunction[16]. Although disease severity and duration highly depend on and influence cytokine levels within the tissue $[8,17]$, circulating cytokines also exacerbate disease severity, increase tissue damage and can even be biomarkers of disease activity [12, 14, 18-20]. Since an association between disease activity and cytokine profiles have been demonstrated, it would be interesting to look at associations between lung involvement and cytokines in patients stratified according to inactive and active disease state. In this setting, the updated PRINTO criteria for clinically inactive disease state in JDM [21] seems promising since they better reflect global disease activity compared to the original criteria [22]. 
Here we aim to explore associations between serum cytokine levels and lung function and structure in a JDM cohort, stratified according to inactive and active disease. Our established Norwegian JDM cohort clinically assessed after median (range)16.8 (6.6-27.0) years of disease duration provide a unique opportunity for this.

\section{PATIENTS AND METHODS}

\section{Patients and controls}

Inclusion criteria were a probable or definitive diagnosis of DM according to the Bohan and Peter criteria [23], disease onset age $<18$ years, disease duration $\geq 24$ month and age $\geq 6$ years at disease onset. 67 patients diagnosed in Norway between January 1970 and June 2006 fulfilled the inclusion criteria. Four were diseased and 62 were tracked through the National Population Register, of whom 59 (95\%) participated in the overall study [7].

Sex and age matched controls were randomly drawn from the National Population Register as previously described in detail [7]. Data on controls including characteristics [7, 15], lung data [7] and cytokine profiles [15] have previously been published. In the present study, data from controls are used to compare correlations between cytokines and pulmonary function in patients with corresponding correlations in the general population.

\section{Data collection and clinical measurement}

All patients and controls were examined at Oslo university hospital (OUS) during a 1-2 day follow up program in the period September 2005 to May 2009, which included clinical examination by a single physician (HS), blood sampling and pulmonary function tests (PFT) and HRCT as described previously [7]. Briefly; disease activity was measured by DAS score for JDM (0-20) and cumulative organ damage by Myositis damage index (MDI)(0-40) [7, 24]. Also 
we present MDI pulmonary damage (called pulmonary damage), which was scored as present (if one of the following items were present: dysphonia, impaired lung function due to respiratory muscle damage, pulmonary fibrosis or pulmonary hypertension) or not present. We defined disease duration as the time from the first skin or muscle symptoms associated with JDM to the time of follow-up examination. History of medication was obtained from the medical records. We used the updated PRINTO criteria to define clinically inactive disease; at least $2 / 3$ of the following: $\mathrm{CK} \leq 150 \mathrm{u} / \mathrm{l}, \mathrm{CMAS} \geq 48, \mathrm{MMT}-8 \geq 78$ and PGA (physician's global assessment of overall disease activity) $\mathrm{PGA} \leq 0.2$ as an essential criterionWe used the updated PRINTO eriterion which includes physician global activity (PGA) as an essential criterion [21] to define clinically inactive disease. This group is defined as JDM-inactive, whereas the remaining patients were defined as JDM-active. All patients are included in JDM-total.

\section{Lung assessments}

PFT and HRCT were performed to obtain lung involvement as previously described [7]. PFT measures are defined as pulmonary function and HRCT as pulmonary structure. In brief the methods were as followed:

PFT were performed on a computerized Vmax Pulmonary Function Unit (Viasys, Santa Ana, California, USA) according to published guidelines [25-27] and included spirometry, gas diffusing capacity and body plethysmography $(\mathrm{n}=55)$ as previously described [7]. For the present study, we used the following variables: forced vital capacity (FVC), diffusing capacity for carbon monoxide (DLCO) (corrected for haemoglobin) and total lung capacity (TLC). The pulmonary function variables were reported as percentage of predicted as previously described. We defined low TLC, DLCO and FVC as less than the fifth percentile of the predicted [28]. 
High-resolution computed tomography (HRCT) was carried out with low dose references in 57 patients using a LightSpeed 16 scanner (GE Healthcare, Milwaukee, Wisconsin, USA). Two patients declined HRCT examination. The images were analyzed by an experienced radiologist blinded to disease characteristics. Established CT criteria for ILD and airways disease were used [29], HRCT-detected ILD was defined as reticular pattern with or_without traction bronchiectasis, and/or ground glass opacity, whereas HRCT-detected airways disease was defined as

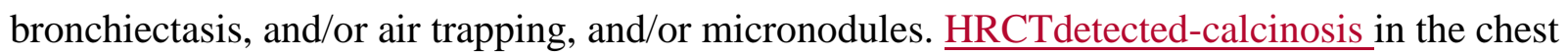
wall (subcutis, fasciae and muscle) was registered and for the present study classified as present or not [7].

\section{Cytokine analysis}

At follow-up, venous blood samples were collected and circulating levels of IL-4, IL-6, IL-8, IL10, IP-10 (CXCL10), MCP-1 (CCL2), macrophage inflammatory protein MIP-1b (CCL4), eotaxin (CCL11), platelet-derived growth factor bb (PDGF) were measured as part of a 27-plex Bio-Plex immunoassay systems (\#m500kcaf0y, Bio-Rad, Hercules, CA), based on xMAP technology (Luminex, Austin,TX). IFN- $\gamma$ and transforming growth factor 1- $\beta$ (TGF1- $\beta$ ) were

analyzed using enzyme linked immunofluorescent assay (ELISA). Both analyses were performed according to manufacturer's protocol, with minor modifications as previously described [15]. Th1/Th2 ratio was estimated from calculation of IFN $\gamma / \mathrm{IL}-4$ levels.

Outlying individuals were found by calculating the mean cytokine levels in JDM-total and the Mahalanobis distance from the cytokine level of each individual to its respective group mean as previously described [15]. One patient and four controls had samples with a p value $<0.001$ and 
were therefore considered to be outliers. These five were removed from the data set before the statistical analyses and data from 58 JDM patients and 56 controls are presented in this study.

\section{Statistics:}

SPSS version 25 (SPSS, Chicago, Il) was used for statistical analyses. Independent samples t-test or chi-square test were used as appropriate, to compare both pulmonary outcomes and cytokine levels between JDM-inactive and JDM-active. Associations between these measures were calculated using Spearmans correlation coefficient $\left(r_{\mathrm{sp}}\right)$ when $n>4$. Strengths of correlations is defined as weak $r_{\mathrm{sp}}=0.1-0.3$, moderate as $\mathrm{r}_{\mathrm{sp}}=0.3-0.6$ and strong $\mathrm{r}_{\mathrm{sp}}=0.6-1.0 . \mathrm{P}$ values $\leq 0.05$ were considered statistically significant. Since age at follow-up might be a possible confounder for associations between cytokines and lung outcomes, we performed correlation analyses between age at follow-up and all presented cytokines; if correlations were $r_{\mathrm{sp}} \geq 0.3$ and statistically significant, the associations between lung outcomes and cytokines in JDM-total were controlled for age in multivariate linear and logistic regression models (using enter), as appropriate. Due to the hypothesis generating nature of our study, we did not correct for multiple comparisons.

\section{Ethic statement:}

Written informed consent were obtained from all patients and controls, and parents if age <16 years, according to the declaration of Helsinki. The study was approved by the Regional Ethics Committee from Medical research (S-05144).

\section{RESULTS}

\section{Characteristics of JDM patients}


Prior to the follow-up visit, 4 were diagnosed with asthma and 7 had dyspnea upon exertion [7] . Three patients had been diagnosed as having ILD. Patient and disease characteristics are shown in table 1. According to the updated PRINTO criteria [21] 21/58 (36.2\%) had inactive disease and 37/58 (63.7\%) had active disease. JDM-active had higher DAS and a trend towards higher MDI than JDM inactive. There was also a trend towards higher MDI pulmonal damage in JDMactive than JDM-inactive.

\section{Lung involvement in JDM patients}

Results of PFT and HRCT findings are shown in table 2. When comparing results of PFT and HRCT in the two groups, we found that DLCO\% predicted was lower in JDM-active than in JDM-inactive. Also there were trends towards poorer lung function in the JDM -active vs JDMinactive patients. The same weak (non-significant) trend, towards more cases of pathologies measured by HRCT was found in JDM-active patients.

\section{Cytokine levels in JDM patients}

Selected cytokines thought to be important for lung involvement either due to associations with lung outcomes in the present data set, or due to known associations in the literature, are shown in table 3. No significant differences between JDM-active and JDM-inactive were found for any cytokines.

\section{Associations between cytokines and lung involvement}

In JDM-total, age at follow-up correlated with eotaxin $\left(\mathrm{r}_{\mathrm{s}}=0.69\right)$ and MCP-1 $\left(\mathrm{r}_{\mathrm{s}}=0.51\right)$, pà $<0.001$ and MIP- $1 \beta\left(\mathrm{r}_{\mathrm{s}}=0.283\right)$ all $\mathrm{p}$ ` $<0.05$. Cytokines with both significant correlations and $\mathrm{r}_{\mathrm{sp}} \geq 0.3$ to pulmonary damage, function or structure in at least one patient group, are presented in table 4 
and visualized in fig.1. Both eotaxin and MCP-1 were associated with MDI-lung-damage in JDM-total and JDM-active patients. These associations were still present after controlling for age in JDM-total (data not shown). The growth factors TGF- $\beta 1$ and PDGF were associated with lower lung volumes (measures of TLC and FVC) in JDM-total, except for no correlation between PDGF and low TLC. PDGF correlated with FVC measures and with low TLC in JDMactive, but not JDM-inactive. On the other hand, TGF- $\beta 1$ correlated with TLC\% predicted and FVC\% predicted in JDM-inactive but not JDM-active. IP-10 was associated with lower TLC\% predicted in active patients only. Higher eotaxin was associated with better gas-diffusion (positive correlation with DLCO\% predicted, and negative association with low DLCO) in JDMtotal and JDM-active. When controlling for age (in JDM-total and JDM-active), eotaxin was no longer significantly associated with DLCO $\%$ predicted or low DLCO $(B=0.009, p=0.70$ and OR 0.99, $\mathrm{p}=0.15$, respectively). MIP-1 $\beta$ was associated with better gas diffusion (DLCO $\%$ predicted and low DLCO) in JDM-inactive patients only. To clarify associations between these cytokines and results of PFT in the general population, the same tests were done for the control group (table 4). No significant correlations were found.

Higher levels of the cytokines IP-10, MCP-1 and eotaxin were associated with HRCT findings in JDM-total and JDM-active, but not in JDM-inactive. IP-10 with any HRCT-findings and HRCT-airways, Eotaxin with HRCT-ILD and MCP-1 with any HRCT-findings (not present in JDM-active) and HRCTdetected-calcinosis. After controlling for age (in JDM-total), the associations between Eotaxin and HRCT ILD and MCP-1 and HRCTdetected-calcinosis was still present whereas the association between MCP-1 and any HRCT findings no longer was significant (data not shown). IL-17 and MIP-1 $\alpha$ were associated with any HRCT findings in JDM-inactive patients only. 


\section{DISCUSSION}

In this study we for the first time show associations between cytokine levels and lung damage, function and structure in JDM patients stratified according to the updated PRINTO criteria for inactive disease. Diffusion capacity was lower in JDM-active than in JDM-inactive patients. IP10, a known biomarker for JDM activity, was found to correlate with total lung capacity and structure in JDM-active patients, and the same structural associations were also found in JDMtotal. Eotaxin was associated with MDI-lung-damage and HRCT-fibrosis, and MCP-1 also with MDI-lung-damage and with any HRCT finding and HRCTdetected-calcinosis in both JDM-total and JDM-active patients.

Regarding representativeness of our study population, all individuals living in Norway have a personal identity number, hence patients diagnosed with JDM in Norway from 1970-2006 could be tracked from the National Registry and 95\% accepted participation. In line with other JDM outcome studies, our cohort has a comparable female predominance and age at onset [2,30]. A larger proportion of our patients had detectable lung involvement compared to another long-term outcome study; however, our patients were more comprehensively examined both regarding PFT in addition to HRCT scans. Also, sex and age matched controls were randomly drawn from the National Registry, which is a strength of our study.

Here we for the first time stratify our Norwegian JDM cohort using the updated PRINTO criteria for inactive disease (which includes PGA as an essential criterion) to evaluate lung outcomes in association with circulating cytokines in JDM patients. Compared to our previous studies using the original PRINTO criteria [24] (known to underestimate non-muscular activity), 6 fewer patients in the present study were defined as inactive. Still, we found no differences between 
JDM-active and JDM-inactive regarding age, disease duration, and anthropometric measures. Naturally, DAS (measuring disease activity in skin and muscle) were higher in JDM-active vs JDM-inactive

As previously reported, the JDM patients mostly had subclinical pulmonary findings, albeit $75 \%$ of the patients showed either functional or structural impairments [7]. Novel findings are that JDM-active had lower diffusion capacity than JDM-inactive. As expected, DLCO implies structural changes in the alveoli, but as in our study, Morganroth et al. reported that $40 \%$ of the DM patients with low DLCO\% predicted showed no signs of ILD [31]. These findings underscore the probability of structural changes occurring which influences DLCO without detectable ILD.

We found no significant difference when comparing cytokines in JDM-active and JDM-inactive patients [21]. These findings are in accordance with an earlier study, in which we used the original PRINTO criteria to stratify patients by disease activity [15].

In our study, IP-10 was the only cytokine being associated with both lower lung volumes in JDM-active and with HRCT findings included airways disease in JDM-total and JDM-active. IP10 has been found in muscle biopsies and suggested upregulated in serum from patients with IIM compared to controls $[32,33]$ and even more important in JDM patients [19]. Also IP-10 is highly associated with disease activity in JDM [19] and progression and severity of ILD in IIM $[18,34]$. In experimental animal models [35, 36] IP-10 induces and enhances pulmonary inflammation and inhibit microvascular remodeling through impaired endothelial cell differentiation and vessel formation [37, 38]. These findings recognize the importance of IP-10 upon inflammation and damage in several areas and tissues of the pulmonary system. In a 
previous study we found lung volumes to be lower in JDM patients with low nail fold capillary density (NCD) compared to those with normal (NCD) [39]. We can hypothesize that IP-10 affects lung volumes by a reduced vascularization in active JDM. Although indirect, this is supported by Jakkula et al. who found that inhibition of vascularization in rat lungs lead to reduced alveolar content and increased alveolar space and hence lower volume [40]. As previously described, TLC\% predicted correlated with both total HRCT abnormalities and HRCT airway [7]. These are the same variables we find IP-10 to inter-correlate with in our study, emphasizing the importance of IP-10 upon pulmonary outcome in JDM. Further MCP-1

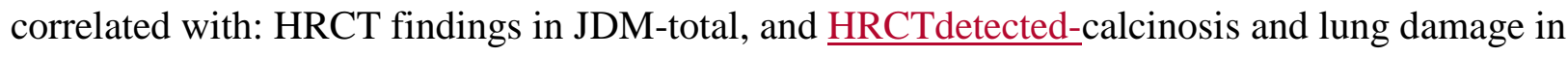
both JDM-total and JDM-active patients. To our knowledge no study has described an association between $\underline{\mathrm{HRCT} d e t e c t e d-c a l c i n o s i s}$ and MCP-1. However, MCP-1 is found to be increased in serum from DM/PM patients with ILD [13], and in bronchoalveolar lavage fluid (BALF) from both children and adults with ILD [41, 42]. Higher levels of lymphocytes correlated with MCP-1and might predict progression and severity of ILD [41, 42]. The inflammatory role of both IP-10 and MCP-1 might be of importance for the structural changes found using HRCT.

Higher eotaxin was associated with more pulmonary damage and HRCT-detected ILD in JDMtotal and in JDM-active patients. To our knowledge eotaxin and pulmonary involvement in related diseases as DM/PM, RA has not been studied. Traditionally eotaxin is associated with asthma and infiltration of eosinophils in the lungs [43]. However, studies from mice reveal an eotaxin dependent lung injury and fibrosis development through infiltration of immune cells in the alveolar spaces [44]. This might be an explanation for the association we find between ILD and eotaxin. To our surprise higher eotaxin was associated with better gas diffusion (DLCO\% 
and low DLCO) in both JDM-total and in JDM-active patients. However, this association was no longer present when controlling for age as a possible confounder. We have previously found an association between eotaxin and age in JDM patients, but not in controls [15]. This is supported by the results from a study by Hossny et al. [45].

MIP-1 $\beta$ was positively associated with DLCO\% in JDM-inactive patients. BALF from patients with pulmonary sarcoidosis contained increased levels of MIP-1 $\beta$ which correlated with inflammatory T-cells, but not with fibrosis related immune cells or DLCO\% predicted [17]. However, eventually these patients developed reduced DLCO\% as in JDM-active patients, suggesting that the inflammatory state of the alveolar membrane is indeed leading to reduced alveolar diffusion. So, the positive correlation between MIP-1 $\beta$ and DLCO\% predicted in JDMinactive is surprising; however, we cannot rule out age as a confounder. MIP-1 $\alpha$ and IL-17 both correlated with any HRCT findings in JDM-inactive patients. MIP-1 $\alpha$ was increased in both alveolar and interstitial immune cells from patients with sarcoidosis or idiopathic pulmonary fibrosis (IPF) compared to healthy controls [46], but associations between MIP-1 $\alpha$, IL-17 and pulmonary findings in IIM has not been studied.

Higher levels of growth factors (TGF- $\beta / \mathrm{PDGF}$ ) were associated with lower lung volumes in JDM-total; PDGF in JDM-active and TGF- $\beta 1$ in JDM-inactive patients. Upon inflammation and damage, PDGF and TGF- $\beta 1$ are mediators of fibrosis in all tissues, altering pulmonary structure and influences lung volumes as pulmonary elasticity is lost [47]. The pathways to fibrosis are complex and differ between the two cytokines, as PDGF induces fibroblast proliferation and migration, whereas TGF- $\beta 1$ activates fibroblast [47]. PDGF and TGF- $\beta 1$ levels in BALF from RA patients have been found to vary with varying severity of ILD. Especially PDGF was elevated in BALF from patients with asymptomatic ILD, but lower in patients with severe IPF 
compared to RA patients without ILD [48]. Also, serum levels of TGF- $\beta 1$ in PM/DM patients with severe ILD were elevated compared to healthy controls [13] hence the growth factors might play a role in different stages of disease activity and in different disease manifestations, as chemokine and immune cell profile alters during disease duration and expression.

Being a cross-sectional study we cannot say anything about the causal effect of the cytokines on lung outcomes. Our results are hypothesis generating, and should be verified, preferable in longitudinal studies. Even though our $\mathrm{n}$ is relatively low, the present study represents the biggest JDM lung outcome study described. Also, our patients were comprehensively phenotyped regarding lung involvement, and we compared results with controls from the general population.

In JDM patients assessed after medium to long-term follow-up, we found a novel association between eotaxin and pulmonary involvement which is not previously described in IIM. Interestingly, we have previously shown an association between eotaxin and both organ damage and cardiac dysfunction in the same cohort. The associations between IP-10, growth factors and MCP-1 with pulmonary involvement are novel in JDM, and have previously been shown in adult onset IIM. The associations were mostly seen in patients with active disease. Further and preferable longitudinal studies are needed no verify our findings.

\section{Figure legends}

Figure 1

\section{Cytokines associated with pulmonary findings in JDM-active and JDM-inactive patients.}

The pulmonary findings: diffusion capacity, structure, volume and damage, and the cytokines 
MIP-1 $\beta$, MIP-1 $\alpha$, IL-17, Eotaxin, MCP-1, IP-10, PDGF and TGF $\beta 1$ are associated as followed: In JDM-active disease increasing eotaxin and MCP-1 are associated with structural alterations and damage, whereas IP-10 is associated with structural changes and declining volumes. The growth factor PDGF seems to affect lung volumes in the same patient group. For the JDMinactive patients: MIP-1 $\beta$ is associated with a better diffusion capacity, MIP-1 $\alpha$ and IL-17 with structural changes and TGF- $\beta 1$ is associated with lower volumes. JDM-active: Italic and underscored.

\section{Funding}

The study was supported by Olav Raagholt and Gerd Meidel Raagholts research foundation and Anders Jahres Fund for promotion of science.

\section{Disclosure statement}

The authors declare no conflict of interest.

\section{Acknowledgements}

Anita Tollisen for help with patient inclusion, Ellen Nordal and Marite Rygg for patient recruitment, Hilde Dishington for laboratory assistance, Maria Vistnes for help with cytokine analysis.

\section{References:}

1. Papadopoulou, C. and L.J. McCann, The Vasculopathy of Juvenile Dermatomyositis. Front Pediatr, 2018. 6: p. 284.

2. Mathiesen, P.R., et al., Pulmonary function and autoantibodies in a long-term follow-up of juvenile dermatomyositis patients. Rheumatology (Oxford), 2014. 53(4): p. 644-9. 
3. Trapani, S., et al., Pulmonary involvement in juvenile dermatomyositis: a two-year longitudinal study. Rheumatology (Oxford), 2001. 40(2): p. 216-20.

4. Kobayashi, I., et al., Interstitial lung disease associated with juvenile dermatomyositis: clinical features and efficacy of cyclosporin A. Rheumatology (Oxford), 2003. 42(2): p. 371-4.

5. Kobayashi, N., et al., Clinical and laboratory features of fatal rapidly progressive interstitial lung disease associated with juvenile dermatomyositis. Rheumatology (Oxford), 2015. 54(5): p. 784-91.

6. Morinishi, Y., et al., Juvenile dermatomyositis: clinical characteristics and the relatively high risk of interstitial lung disease. Mod Rheumatol, 2007. 17(5): p. 413-7.

7. Sanner, H., et al., Pulmonary outcome in juvenile dermatomyositis: a case-control study. Ann Rheum Dis, 2011. 70(1): p. 86-91.

8. Gono, T., et al., Cytokine profiles in polymyositis and dermatomyositis complicated by rapidly progressive or chronic interstitial lung disease. Rheumatology (Oxford), 2014. 53(12): p. 2196203.

9. $\quad \mathrm{Li}, \mathrm{C} . \mathrm{K}$. , et al., MHC Class I overexpression on muscles in early juvenile dermatomyositis. $\mathrm{J}$ Rheumatol, 2004. 31(3): p. 605-9.

10. Arshanapalli, A., et al., The role of type I interferons and other cytokines in dermatomyositis. Cytokine, 2015. 73(2): p. 319-25.

11. Reed, A.M., et al., Changes in novel biomarkers of disease activity in juvenile and adult dermatomyositis are sensitive biomarkers of disease course. Arthritis Rheum, 2012. 64(12): p. 4078-86.

12. Wienke, J., et al., Galectin-9 and CXCL10 as biomarkers for disease activity in juvenile dermatomyositis: a longitudinal cohort study and multi-cohort validation. Arthritis Rheumatol, 2019.

13. Wu, C.Y., L. Li, and L.H. Zhang, Detection of serum MCP-1 and TGF-betal in polymyositis/dermatomyositis patients and its significance. Eur J Med Res, 2019. 24(1): p. 12.

14. Bilgic, H., et al., Interleukin-6 and type I interferon-regulated genes and chemokines mark disease activity in dermatomyositis. Arthritis Rheum, 2009. 60(11): p. 3436-46.

15. Sanner, H., et al., Increased levels of eotaxin and MCP-1 in juvenile dermatomyositis median 16.8 years after disease onset; associations with disease activity, duration and organ damage. PLoS One, 2014. 9(3): p. e92171.

16. Schwartz, T., et al., In active juvenile dermatomyositis, elevated eotaxin and MCP-1 and cholesterol levels in the upper normal range are associated with cardiac dysfunction. Rheumatology (Oxford), 2014. 53(12): p. 2214-22.

17. Capelli, A., et al., Increased macrophage inflammatory protein-1alpha and macrophage inflammatory protein-1beta levels in bronchoalveolar lavage fluid of patients affected by different stages of pulmonary sarcoidosis. Am J Respir Crit Care Med, 2002. 165(2): p. 236-41.

18. Chen, J., et al., Biomarkers of rheumatoid arthritis-associated interstitial lung disease. Arthritis Rheumatol, 2015. 67(1): p. 28-38.

19. Bellutti Enders, F., et al., Correlation of CXCL10, tumor necrosis factor receptor type II, and galectin 9 with disease activity in juvenile dermatomyositis. Arthritis Rheumatol, 2014. 66(8): $\mathrm{p}$. 2281-9.

20. Nasr, R., A.M. Reed, and E.J. Peterson, Update: biomarkers for idiopathic inflammatory myopathies. Curr Opin Rheumatol, 2012. 24(6): p. 609-15.

21. Almeida, B., et al., Analysis of Published Criteria for Clinically Inactive Disease in a Large Juvenile Dermatomyositis Cohort Shows That Skin Disease Is Underestimated. Arthritis Rheumatol, 2015. 67(9): p. 2495-502.

22. Lazarevic, D., et al., The PRINTO criteria for clinically inactive disease in juvenile dermatomyositis. Ann Rheum Dis, 2013. 72(5): p. 686-93.

23. Bohan, A. and J.B. Peter, Polymyositis and dermatomyositis (first of two parts). N Engl J Med, 1975. 292(7): p. 344-7. 
24. Rider, L.G., et al., Measures of adult and juvenile dermatomyositis, polymyositis, and inclusion body myositis: Physician and Patient/Parent Global Activity, Manual Muscle Testing (MMT), Health Assessment Questionnaire (HAQ)/Childhood Health Assessment Questionnaire (C-HAQ), Childhood Myositis Assessment Scale (CMAS), Myositis Disease Activity Assessment Tool (MDAAT), Disease Activity Score (DAS), Short Form 36 (SF-36), Child Health Questionnaire (CHQ), physician global damage, Myositis Damage Index (MDI), Quantitative Muscle Testing (QMT), Myositis Functional Index-2 (FI-2), Myositis Activities Profile (MAP), Inclusion Body Myositis Functional Rating Scale (IBMFRS), Cutaneous Dermatomyositis Disease Area and Severity Index (CDASI), Cutaneous Assessment Tool (CAT), Dermatomyositis Skin Severity Index (DSSI), Skindex, and Dermatology Life Quality Index (DLQI). Arthritis Care Res (Hoboken), 2011. 63 Suppl 11: p. S118-57.

25. Macintyre, N., et al., Standardisation of the single-breath determination of carbon monoxide uptake in the lung. Eur Respir J, 2005. 26(4): p. 720-35.

26. Miller, M.R., et al., Standardisation of spirometry. Eur Respir J, 2005. 26(2): p. 319-38.

27. Wanger, J., et al., Standardisation of the measurement of lung volumes. Eur Respir J, 2005. 26(3): p. 511-22.

28. Loetscher, P., et al., The ligands of CXC chemokine receptor 3, I-TAC, Mig, and IP10, are natural antagonists for CCR3. J Biol Chem, 2001. 276(5): p. 2986-91.

29. Austin, J.H., et al., Glossary of terms for CT of the lungs: recommendations of the Nomenclature Committee of the Fleischner Society. Radiology, 1996. 200(2): p. 327-31.

30. Constantin, T., et al., National registry of patients with juvenile idiopathic inflammatory myopathies in Hungary--clinical characteristics and disease course of 44 patients with juvenile dermatomyositis. Autoimmunity, 2006. 39(3): p. 223-32.

31. Morganroth, P.A., et al., Interstitial Lung Disease in Classic and Skin-Predominant Dermatomyositis: A Retrospective Study With Screening Recommendations. Archives of Dermatology, 2010. 146(7): p. 729-738.

32. De Paepe, B., et al., Alpha-chemokine receptors CXCRI-3 and their ligands in idiopathic inflammatory myopathies. Acta Neuropathol, 2005. 109(6): p. 576-82.

33. Uruha, A., et al., Plasma IP-10 level distinguishes inflammatory myopathy. Neurology, 2015. 85(3): p. 293-4.

34. Richards, T.J., et al., Characterization and peripheral blood biomarker assessment of anti-Jo-1 antibody-positive interstitial lung disease. Arthritis Rheum, 2009. 60(7): p. 2183-92.

35. Dixon, A.E., et al., Chemokine expression in Th1 cell-induced lung injury: prominence of IFNgamma-inducible chemokines. Am J Physiol Lung Cell Mol Physiol, 2000. 279(3): p. L592-9.

36. Gao, B., et al., Upregulation of chemokine CXCL10 enhances chronic pulmonary inflammation in tree shrew collagen-induced arthritis. Sci Rep, 2018. 8(1): p. 9993.

37. Angiolillo, A.L., et al., Human interferon-inducible protein 10 is a potent inhibitor of angiogenesis in vivo. J Exp Med, 1995. 182(1): p. 155-62.

38. Luster, A.D., et al., Delayed wound healing and disorganized neovascularization in transgenic mice expressing the IP-10 chemokine. Proc Assoc Am Physicians, 1998. 110(3): p. 183-96.

39. Barth, Z., et al., The Association Between Nailfold Capillary Density and Pulmonary and Cardiac Involvement in Medium- to Long-standing Juvenile Dermatomyositis. Arthritis Care Res (Hoboken), 2018.

40. Jakkula, M., et al., Inhibition of angiogenesis decreases alveolarization in the developing rat lung. Am J Physiol Lung Cell Mol Physiol, 2000. 279(3): p. L600-7.

41. Suga, M., et al., Clinical significance of MCP-1 levels in BALF and serum in patients with interstitial lung diseases. Eur Respir J, 1999. 14(2): p. 376-82.

42. Hartl, D., et al., A role for MCP-1/CCR2 in interstitial lung disease in children. Respir Res, 2005. 6: p. 93.

43. Conroy, D.M. and T.J. Williams, Eotaxin and the attraction of eosinophils to the asthmatic lung. Respir Res, 2001. 2(3): p. 150-6. 
44. Huaux, F., et al., Role of Eotaxin-1 (CCL11) and CC chemokine receptor 3 (CCR3) in bleomycininduced lung injury and fibrosis. Am J Pathol, 2005. 167(6): p. 1485-96.

45. Hossny, E., M. Aboul-Magd, and S. Bakr, Increased plasma eotaxin in atopic dermatitis and acute urticaria in infants and children. Allergy, 2001. 56(10): p. 996-1002.

46. Standiford, T.J., et al., Macrophage inflammatory protein-1 alpha expression in interstitial lung disease. J Immunol, 1993. 151(5): p. 2852-63.

47. Bonner, J.C., Regulation of PDGF and its receptors in fibrotic diseases. Cytokine Growth Factor Rev, 2004. 15(4): p. 255-73.

48. Gochuico, B.R., et al., Progressive preclinical interstitial lung disease in rheumatoid arthritis. Arch Intern Med, 2008. 168(2): p. 159-66. 
Table 1. Characteristics, disease variables in JDM patients

\begin{tabular}{lllll}
\hline & $\begin{array}{l}\text { JDM-tot } \\
(\mathbf{n = 5 8})\end{array}$ & $\begin{array}{l}\text { JDM-active } \\
(\mathbf{n = 3 7})\end{array}$ & $\begin{array}{l}\text { JDM-inactive } \\
(\mathbf{n}=\mathbf{2 1})\end{array}$ & p value \\
\hline Age, y (range) & $20.9(6.7-55.4)$ & $19.8(6.7-55.4)$ & $25.7(11.8-43.3)$ & 0.39 \\
Female & $35(59.3 \%)$ & $25(67.6 \%)$ & $10(47.6 \%)$ & 0.14 \\
Disease duration, $\mathbf{y}$ & $16.8(6.6-27.0)$ & $15.6(5.7-26.3)$ & $17.1(8.2-27.5)$ & 0.34 \\
Height $(\mathbf{c m})$ & $166(15)$ & $163(17)$ & $170(9)$ & 0.07 \\
Weight $(\mathbf{k g})$ & $62.8(20.2)$ & $60.8(22.7)$ & $66.3(14.6)$ & 0.33 \\
BMI $\left(\mathbf{k g} / \mathbf{m}^{2}\right)$ & $22.3(4.8)$ & $22.1(5.1)$ & $22.7(4.3)$ & 0.65 \\
DAS $(\mathbf{0 - 2 0})$ & $4.75(2.8-6.0)$ & $6(4.5-7.3)$ & $2(0.0-4.0)$ & $<0.001 * *$ \\
MDI $(\mathbf{0 - 4 0 )}$ & $3(2.0-6.0)$ & $5(2.0-7.0)$ & $3(1.0-5.0)$ & 0.07 \\
MDI lung damage present, $\mathbf{n}(\boldsymbol{\%})$ & $10(16.9)$ & $8(21.6)$ & $2(9.5)$ & 0.25 \\
Use of medication at follow up, $\mathbf{n}(\boldsymbol{\%})$ & $16(29.6)$ & $15(40.5)$ & $1(4.8)$ & $0.002 * *$ \\
\hline $\begin{array}{l}\text { BMI: Body mass index, DAS: Disease activity score, MDI: Myositis damage index. Values are median }(\mathrm{IQR}), \mathrm{n}(\%) \\
\text { or mean }(\mathrm{SD}) * \mathrm{p}<0.05, * * \mathrm{p}<0.01 \text { when comparing active vs inactive }\end{array}$
\end{tabular}


Table 2. Pulmonary findings in JDM patients

\begin{tabular}{lllll}
\hline Function & $\begin{array}{l}\text { JDM-tot } \\
\mathbf{n}=(\mathbf{5 7 - 5 8})\end{array}$ & $\begin{array}{l}\text { JDM-active } \\
(\mathbf{n}=\mathbf{3 7})\end{array}$ & $\begin{array}{l}\text { JDM-inactive } \\
(\mathbf{n = 2 1})\end{array}$ & p value \\
\hline TLC\% & $92.5(10.5)$ & $91.0(10.3)$ & $94.3(10.8)$ & 0.30 \\
Low TLC & $14(23.7)$ & $10(26.3)$ & $4(19.0)$ & 0.36 \\
FVC \% & $95.8(12.4)$ & $93.9(12.3)$ & $98.7(12.1)$ & 0.19 \\
Low FVC & $10(16.9)$ & $8(21.1)$ & $2(9.5)$ & 0.24 \\
DLCO \% & $81.6(14.8)$ & $78.6(13.0)$ & $87.4(16.2)$ & $0.03^{*}$ \\
Low DLCO & $29(49.2)$ & $21(55.3)$ & $8(38.1)$ & 0.17 \\
HRCT,any changes & $21(35.6)$ & $15(39.5)$ & $6(28.6)$ & 0.39 \\
HRCT,ILD/fibrosis & $8(13.6)$ & $6(15.8)$ & $2(9.5)$ & 0.50 \\
HRCT,calcinosis & $8(13.6)$ & $6(15.8)$ & $2(9.5)$ & 0.50 \\
HRCT,airways & $9(15.3)$ & $7(18.4)$ & $2(9.5)$ & 0.36 \\
\hline
\end{tabular}

TLC: Total lung capacity, FVC: forced vital capacity, DLCO: diffusing capacity of the lung for carbon monoxide, HRCT: High resolution computed tomography, ILD: Interstitial lung disease. Values are mean (SD) or number (\%); p-value: when comparing active vs inactive 
Table 3. Cytokine levels in JDM patients;

\begin{tabular}{|c|c|c|c|c|}
\hline Cytokines & $\begin{array}{l}\text { JDM-tot } \\
(\mathrm{n}=57)\end{array}$ & $\begin{array}{l}\text { JDM-active } \\
(\mathbf{n}=37)\end{array}$ & $\begin{array}{l}\text { JDM-inactive } \\
(n=21)\end{array}$ & p value \\
\hline IFN $\gamma$ & $55.1(69.0)$ & $62.2(84.2)$ & $41.8(24.7)$ & 0.27 \\
\hline Th1/Th2 & $25.4(28.6)$ & $28.6(35.0)$ & $19.8(8.7)$ & 0.24 \\
\hline TNFa & $22.2(21.4)$ & $24.8(26.0)$ & $17.8(7.9)$ & 0.24 \\
\hline MIP-1a & $2.5(1.4)$ & $2.7(1.5)$ & $2.3(1.1)$ & 0.38 \\
\hline MIP-1及 & $104.4(42.3)$ & $102.4(37.1)$ & $108.0(50.9)$ & 0.64 \\
\hline PDGF & 9083 (2799) & 8990 (2979) & $9248(2511)$ & 0.74 \\
\hline TGF- $\beta 1$ & 29252 (8868) & 29698 (9623) & 28467 (7513) & 0.62 \\
\hline MCP-1 & $34.0(21.3)$ & $34.3(18.8)$ & $33.5(25.6)$ & 0.89 \\
\hline Eotaxin & $138(103)$ & $142(108)$ & $132(95)$ & 0.72 \\
\hline IP-10 & $1448(1281)$ & 1491 (1438) & $1371(972)$ & 0.74 \\
\hline IL-6 & $6.7(10.2)$ & $7.8(12.5)$ & $4.4(2.9)$ & 0.24 \\
\hline IL-4 & $2.1(0.54)$ & $2.1(0.59)$ & $2.1(0.45)$ & 0.79 \\
\hline IL-8 & $10.9(3.0)$ & $10.8(3.1)$ & $11.05(2.7)$ & 0.81 \\
\hline IL-10 & $4.8(16.7)$ & $6.1(20.8)$ & $2.4(2.2)$ & 0.42 \\
\hline IL-17 & $15.1(13.5)$ & $15.0(15.1)$ & $15.2(10.4)$ & 0.97 \\
\hline
\end{tabular}

IFN $\gamma$ : Interferon gamma, TNF $\alpha$ : Tumor necrosis factor alpha, MIP-1 $\alpha$ and $\beta$ : Macrophage inflammatory protein 1alpha and beta, PDGF: Platelet-derived growth factor, TGF $\beta 1$ : Transforming growth factor beta 1, MCP-1/CCL2: Monocyte chemoattractant protein 1, IP-10/CXCL10: IFN- $\gamma$-inducible protein 10, IL: Interleukin. Values are mean (SD) $\mathrm{pg} / \mathrm{ml}$; $\mathrm{p}$-value when comparing active vs inactive 
Table 4. Associations between pulmonary findings and cytokines in JDM patients and controls.

\begin{tabular}{|c|c|c|c|c|c|}
\hline & & $\begin{array}{c}\text { JDM-total } \\
(\mathrm{n}=50-54)\end{array}$ & $\begin{array}{c}\text { JDM-active } \\
(\mathrm{n}=30-34)\end{array}$ & $\begin{array}{c}\text { JDM-inactive } \\
(\mathrm{n}=20)\end{array}$ & $\begin{array}{r}\text { Controls } \\
(\mathrm{n}=51-56)\end{array}$ \\
\hline \multirow[t]{3}{*}{ Damage } & \multicolumn{5}{|c|}{ MDI lung damage present } \\
\hline & Eotaxin & $0.41 * *$ & $0.49 * *$ & NA & NA \\
\hline & MCP-1 & $0.44 * *$ & $0.42 * *$ & NA & NA \\
\hline \multirow[t]{21}{*}{ Function } & \multicolumn{5}{|c|}{ Lung volume: Total Lung Capacity (TLC) } \\
\hline & \multicolumn{5}{|c|}{ TLC\% } \\
\hline & IP-10 & -0.14 & $-0.35^{*}$ & 0.19 & 0.03 \\
\hline & PDGF & $-0.33 *$ & -0.31 & -0.43 & 0.05 \\
\hline & TGF- $\beta 1$ & $-0.30^{*}$ & -0.15 & $-0.62 * *$ & 0.01 \\
\hline & LOW TLC & & & & \\
\hline & PDGF & 0.26 & $0.35 *$ & NA & -0.05 \\
\hline & TGF- $\beta 1$ & $0.30^{*}$ & 0.31 & NA & 0.04 \\
\hline & \multicolumn{5}{|c|}{ Lung volume: Forced Vital Capacity (FVC) } \\
\hline & \multicolumn{5}{|c|}{ FVC\% } \\
\hline & PDGF & $-0.38 * *$ & $-0.48 * *$ & NA & -0.05 \\
\hline & TGF- $\beta 1$ & $-0.33^{*}$ & -0.20 & $-0.59 * *$ & -0.10 \\
\hline & LOW FVC & & & & \\
\hline & PDGF & $0.33 *$ & $0.44 * *$ & 0.08 & 0.02 \\
\hline & \multicolumn{5}{|c|}{ Alveolar function: Diffusion Capacity (DLCO) } \\
\hline & \multicolumn{5}{|c|}{ DLCO\% } \\
\hline & MIP-1 $\beta$ & 0.13 & -0.01 & $0.47 *$ & 0.04 \\
\hline & Eotaxin & $0.39 * *$ & $0.52 * *$ & 0.1 & $0.28 *$ \\
\hline & LOW DLCO & & & & \\
\hline & MIP-1 $\beta$ & -0.14 & 0.03 & $-0.44 *$ & -0.02 \\
\hline & Eotaxin & $-0.32 *$ & $-0.53 * *$ & 0.01 & -0.24 \\
\hline \multirow[t]{12}{*}{ Structure } & \multicolumn{5}{|c|}{ High Resolution Computer Tomography (HRCT) } \\
\hline & \multicolumn{5}{|c|}{ Any HRCT findings } \\
\hline & IP-10 & $0.37 * *$ & $0.34 *$ & NA & NA \\
\hline & MCP-1 & $0.34 * *$ & 0.32 & NA & NA \\
\hline & MIP- $1 \alpha$ & 0.13 & -0.1 & $0.63 * *$ & NA \\
\hline & IL-17 & 0.06 & -0.16 & $0.61 * *$ & NA \\
\hline & \multicolumn{5}{|l|}{ HRCT ILD } \\
\hline & Eotaxin & $0.37 * *$ & $0.38^{*}$ & NA & NA \\
\hline & \multicolumn{5}{|l|}{ HRCT airways } \\
\hline & IP-10 & $0.44 * *$ & $0.61 * *$ & NA & NA \\
\hline & \multicolumn{5}{|l|}{ HRCT calcinosis } \\
\hline & MCP-1 & $0.40 * *$ & $0.34 *$ & NA & NA \\
\hline
\end{tabular}

IP-10/CXCL10: Interferon gamma inducible protein 10, PDGF: Platelet-derived growth factor, TGF $\beta 1$ :

Transforming growth factor beta-1, MIP- $1 \alpha$ and $1 \beta$ : Macrophage inflammatory protein alpha and beta, MCP-1: Monocyte chemoattractant protein, IL-17: Interleukin 17, ILD: Interstitial lung disease, MDI: myositis damage index, NA: non-applicable. All values are Spearmans correlation. * $\mathrm{p}<0.05, * * \mathrm{p}<0.01$ 
Figure 1

Cytokines associated with pulmonary findings in JDM patients

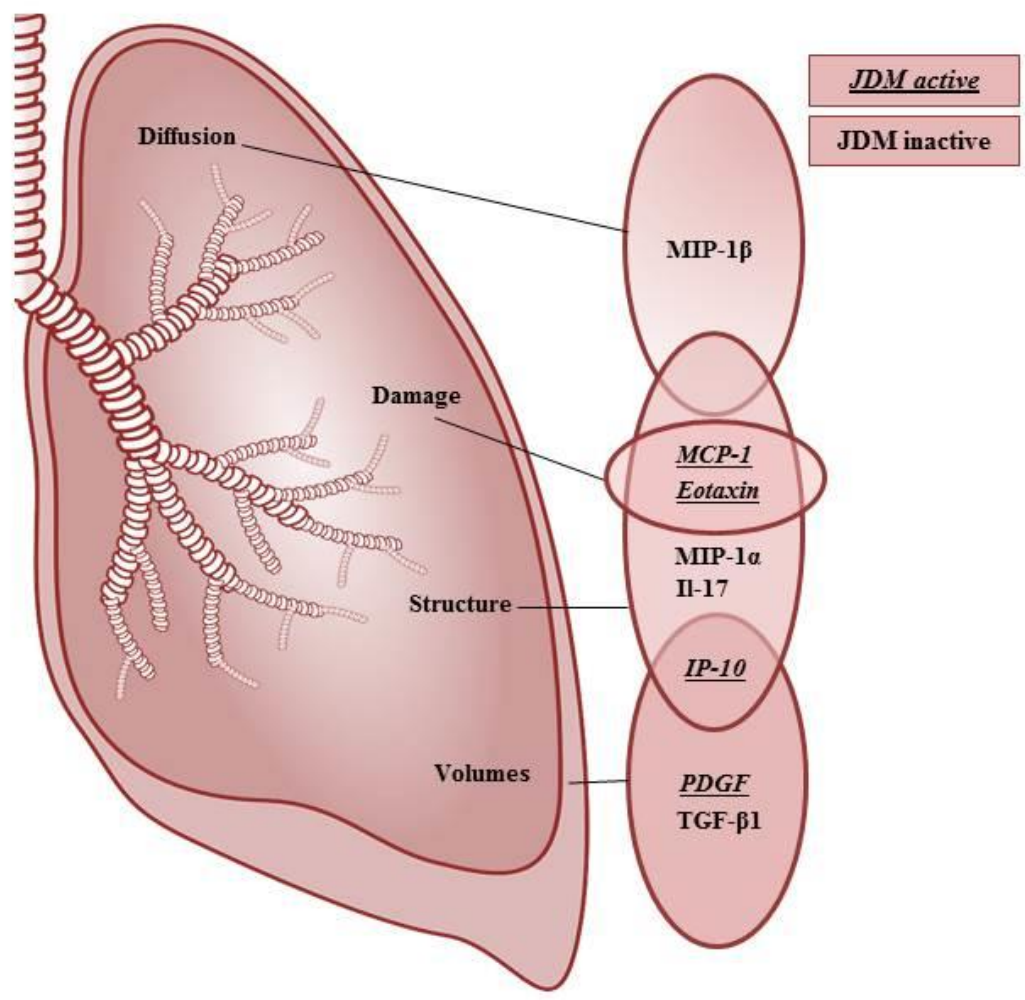

